\title{
Parameters for the Generalized Born Model Consistent with RESP Atomic Partial Charge Assignment Protocol
}

\author{
Wei Zhang, Tingjun Hou, Xuebin Qiao, and Xiaojie $\mathrm{Xu}^{*}$ \\ College of Chemistry and Molecules Engineering, Peking University, Beijing 100871, China
}

Received: March 11, 2003; In Final Form: May 16, 2003

\begin{abstract}
Here we report a set of new parameters for the generalized Born (GB) model consistent with the RESP atomic partial charge assignment protocol. Effective atomic radii and screen factors as parameters have been obtained through genetic algorithm optimization in the parameter space to minimize the differences between the calculated and experimental solvation free energies. Here, the calculated solvation free energies are based on a GB model using partial charges fitted from the electrostatic potentials based on the $6-31 \mathrm{G}^{*}$ basis set with the nonelectrostatic contributions to the free energy of solvation modeled in terms of the solvent accessible surface area (SASA). The mean unsigned error in the solvation free energies calculated by the GB/surface area calculations using the final parameters of the 328 neutral molecules in the training set is $0.85 \mathrm{kcal} / \mathrm{mol}$, and for the 30 charged molecules the value is $4.36 \mathrm{kcal} / \mathrm{mol}$. The refined parameters were then applied to predict the solvation free energies of 44 neutral or charged organic molecules and 15 proteins, and reliable results were obtained for both organic molecules and proteins. For the 36 neutral organic molecules in the test set, our parameters incurred an unsigned mean error of $0.73 \mathrm{kcal} / \mathrm{mol}$, and for the eight charged molecules in the test set, our parameters incurred an unsigned mean error of $3.65 \mathrm{kcal} / \mathrm{mol}$. For the 44 organic molecules, the performance of the GB/SA model based on our new parameters was much better than Possion-Boltzmann (PB)/SA and GB/SA based on Jayaram's parameters. For the 15 proteins randomly selected from the Protein Data Bank, the calculated results from GB/SA based on our new parameters also gave consistent results with those from $\mathrm{PB} / \mathrm{SA}$ and were much better than GB/SA based on Jayaram's parameters. This model might be widely applied in molecules dynamics, protein folding, molecular docking, free energy calculations, and conformation analysis. Moreover, we are now supplying a program to help AMBER users apply our new parameters to their MD simulations.
\end{abstract}

\section{Introduction}

Accurately and rapidly modeling solvation is crucial to quantitatively understanding the chemical and physical properties that underly many biochemical processes. To solve this problem, both molecules ${ }^{1-3}$ and continuum ${ }^{4-10}$ models of solvent have been developed. Explicit solvent models employ thousands of discrete solvent molecules and have been widely used for simulations in the liquid environment. Many properties of the solutions can be reproduced by calculations employing explicit solvent models, but such calculations converge very slowly because of the large number of particles and states involved. Because explicit solvent models are so computationally demanding, there is interest in developing more rapid continuum solvation models. Continuum solvation models treat the solvent as a continuous medium surrounding the solute beginning near its van der Waals surface. In principle, such models can predict solvation effects with relatively little computational resources, because the model includes no particles other than the atoms of solute.

A lot of continuum solvation models have been reported over the years. Many treatments of these were based on the surface area (SA) or solvent accessible surface area (SASA). ${ }^{1-15}$ However, we are concerned that area-based representations provide poor approximations for the long-range electrostatic component of solvation. Another popular approach to continuum

* Corresponding author. E-mail: xiaojxu@chem.pku.edu.cn. solvation treats the solvent as a high dielectric continuum, interacting with charges that are embedded in solute molecules of lower dielectric media. ${ }^{16-18}$ Despite the severity of the approximation, this model gives a good account of the electrostatic component of solvation energy. However, such dielectric continuum models of the solvent do not include van der Waals solvent-solute interaction terms.

Because of the shortcomings of the previous models, the Possion-Boltzmann (PB)/SA model and the generalized Born (GB)/SA model had been developed, which provided solvation free energy based on the PB equation ${ }^{19}$ or $\mathrm{GB}$ model $^{9}$ for the electrostatic component and SA for the nonpolar component of the solvation free energy.

In the $\mathrm{PB} / \mathrm{SA}$ and $\mathrm{GB} / \mathrm{SA}$ models, the solvation free energy is given as the sum of the solute-solvent van der Waals term and the solute-solvent electrostatic polarization term, as illustrated in eq 1 .

$$
G_{\mathrm{sol}}=G_{\mathrm{vdw}}+G_{\mathrm{pol}}
$$

Because hydrocarbons are nonpolar molecules $\left(G_{\mathrm{pol}} \sim 0\right)$, and their $G_{\text {sol }}$ in water is approximately linearly related ${ }^{13-15}$ to their SASA, we compute the solute-solvent van der Waals term by evaluating the SASA.

$G_{\mathrm{pol}}$ are usually presented by the PB equation, which is typically solved by finite-difference or boundary element numerical methods. ${ }^{8,17-19}$ The equation solving procedure may become very expensive for proteins and nucleic acids, so there 
is a interest in finding an approximate solvation for the PB equation. One candidate is the GB approach. ${ }^{9}$ In this model, the polar term of solvation free energy is represented by eqs 2 and 3 .

$$
\begin{aligned}
& \Delta G_{\mathrm{pol}}=-\frac{1}{2}\left(1-\frac{1}{\epsilon_{\mathrm{w}}}\right) \sum_{i=1}^{N} \sum_{j=1}^{N} \frac{q_{i} q_{j}}{f_{\mathrm{GB}}} \\
& f_{\mathrm{GB}}=\sqrt{r_{i j}{ }^{2}+R_{i} R_{j} \exp \left(-r_{i j}{ }^{2} / 4 R_{i} R_{j}\right)}
\end{aligned}
$$

Among the equations, $q_{i}$ and $q_{j}$ is the partial charge of atoms $i$ and $j$, respectively; $\epsilon_{\mathrm{w}}$ is the solvent dielectric constant of the media; $r_{i j}$ is the distance between atoms $i$ and $j$; and $R_{i}$ and $R_{j}$ is the effective Born radii of atoms $i$ and $j$, respectively. In its original form, $R_{i}$ was estimated by a numerical integration procedure, but recently a pairwise approximation calculation of effective Born radii has been reported, and was widely accepted in estimating the solvation free energies of proteins. ${ }^{20}$ The continuum solvation model has been reviewed heavily in the recent past, to include GB models. ${ }^{21,22} \mathrm{~A}$ nice review of both continuum and explicit models applied to biological problems is that by Orozco and Luque. ${ }^{23}$

The energy calculations of protein and nucleic acids are typically based on force fields such as AMBER, CHARMM, and GROMOS, and each force field has its own rule in determining the atomic partial charges. For instance, the AMBER 6.0 suite software package employs a so-called RESP atomic partial charge assignment protocol, so for GB models run on these force fields, it is necessary to determine a set of parameters to estimate effective Born radius accurately. In 1998, Jayaram et al. reported a set of parameters for GB models consistent with the RESP protocol. ${ }^{24}$ In 2000, Cheng et al. reported a set of parameters for GB models consistent with the MMFF force field. ${ }^{25}$ Recently, Liu et al. reported GB parameters consistent with the GROMOS 96 force field. ${ }^{26}$

As we mentioned above, there was already a set of GB parameters consistent with the RESP protocol reported by Jayaram et al. in 1998, but their parameters were designed only for proteins, and not suitable for studying the protein-inhibitor complex system; so for many organic molecules, the performance of GB/SA based on the Jayaram's parameters is very poor. Moreover, Jayaram's group employed a relatively small training set (32 molecules) to derive their parameters, which is insufficient for dealing with small organic molecule systems, so there is interest in a set of GB parameters that could predict the solvation free energy of small organic molecules accurately.

Here we report a set of parameters for the GB model consistent with the RESP atomic partial charge assignment protocol. This set of parameters was derived by fitting to the experimental solvation free energies of 358 small organic molecules. To our knowledge, the training set used here is significantly larger than those in previous work. ${ }^{24-26}$ The adoption of a large training set gives us more opportunity to define more elaborate definitions of atom typing rules. Furthermore, we used the genetic algorithm (GA) to optimize the difference between the calculated solvation free energies and the experimental solvation free energies. As a very efficient stochastic optimization method, it has been widely used to solve the minimization problems such as conformational search, ${ }^{27}$ molecular docking, ${ }^{28-30}$ and QSAR. ${ }^{31-33} \mathrm{We}$ expect that by applying GA as the optimization method, the parameters for each atom type should achieve the most optimal values. The Minnesota group has used GA to develop all of their quantum mechanical GB models (the SMx models) and have gotten brilliant results. ${ }^{34}$

\section{Methodology}

Data Set. We selected 402 organic molecules to perform the parametrization. Their names and experimental solvation free energies are included in Supporting Information. The experimental solvation free energies were determined at $298 \mathrm{~K}, 1 \mathrm{~atm}$. The molecular geometries of all compounds were modeled using the Cerius ${ }^{2}$ molecular simulation package. ${ }^{35}$ The initial structures were fully minimized using molecular mechanics with the MMFF force field. ${ }^{36}$ Conformational analyses were performed for some molecules with flexible chains to find the global minimum geometries. For each molecule, only the global minimum conformation was used in the subsequent parametrization. The whole data set was divided into a training set with 358 molecules and a test set with 44 molecules. For all molecules in the training set, 328 molecules were neutral, while the other 30 molecules were charged. The solvation parameters were determined based on the training set, and the actual prediction ability was validated by the test set. The parametrization procedure is basically searching for a set of parameters that could reproduce the solvation free energies of the parametrization set molecules. The electrostatic contribution to solvation free energy can be estimated according to eqs 2 and 3, which requires an input of atomic partial charge, atomic Cartesian coordinates, and atomic initial Born radii. The nonpolar contribution to the solvation free energy is estimated by a molecule's SASA. The derived model was then applied to predict the solvation free energy of two test sets.

Among the first test set made up of 44 simple organic molecules, 36 molecules were neutral and 8 molecules were charged. As the second test set, 15 proteins were chosen randomly from the Brookhaven Protein Data Bank (PDB). For these proteins, all crystallographic water molecules were eliminated from the structures. Some missing hydrogen atoms were added using the InsightII molecular simulation package, ${ }^{37}$ with a neutral sp3 N-terminus and a carboxylic $(\mathrm{COOH})$ $\mathrm{C}$-terminus assigned at neutral $\mathrm{pH}$. These structures were minimized using the AMBER force field to remove any steric overlap with a restraint of the main chain.

Atom Typing Rule. We classified the atoms in molecules according to its element and hybridization. A total of 21 atom types were introduced, and four of them were designed for representing charged molecules. The definition of these atom types was based on the SMARTS description (see Table 1). ${ }^{38}$ SMARTS is a language that allows you to specify substructures using rules that are straightforward extensions of SMILES. In fact, almost all SMILES specifications are valid SMARTS targets. As SMILES, in SMARTS one can use atomic and bond symbols to specify a graph. However, in SMARTS the labels for the graph's nodes and edges (its "atoms" and "bonds") are extended to include "logical operators" and special atomic and bond symbols; these allow SMARTS atoms and bonds to be more general. Using SMARTS, flexible and efficient substructure search specifications can be made in terms that are meaningful to chemists. In the current work, a parameter file was used to store the SMARTS chains defined for all atom types. If we want to add some new typing rules or modify the typing rules, we only need to make some modifications to this parameter file.

Derivation of Atomic Coordinate and Atomic Partial Charges. Partial charges were derived to be consistent with the AMBER charge derivation protocols. All the studied organic 
TABLE 1: Typing Protocol of Atom Types and Derived Parameters for Generalized Born Solvation Model

\begin{tabular}{|c|c|c|c|c|c|}
\hline no. & name & description & $\begin{array}{c}\text { occurrence in } \\
\text { parametrization set }\end{array}$ & radii & $\begin{array}{c}\text { screen } \\
\text { parameter }\end{array}$ \\
\hline 1 & $\mathrm{HC}$ & hydrogen atom connected to alkane carbon & 2707 & 1.6 & 0.5 \\
\hline 2 & $\mathrm{H} 1$ & hydrogen atom connected to polar atom & 100 & 0.9 & 1.2 \\
\hline 3 & HA & hydrogen atom connected to aromatic carbon & 376 & 0.9 & 0.8 \\
\hline 4 & $\mathrm{C} 1$ & $\mathrm{sp}^{1}$ carbon atom & 25 & 2.5 & 0.9 \\
\hline 5 & $\mathrm{C} 2$ & $\mathrm{sp}^{2}$ carbon atom & 155 & 2.4 & 0.5 \\
\hline 6 & $\mathrm{C} 3$ & $\mathrm{sp}^{3}$ carbon atom & 1151 & 2.1 & 0.6 \\
\hline 7 & $\mathrm{CA}$ & aromatic carbon & 538 & 1.7 & 0.8 \\
\hline 8 & N1 & $\mathrm{sp}^{1}$ nitrogen atom & 7 & 1.4 & 1.5 \\
\hline 9 & N2 & $\mathrm{sp}^{2}$ nitrogen atom & 51 & 1.3 & 0.7 \\
\hline 10 & N3 & $\mathrm{sp}^{2}$ nitrogen atom & 28 & 1.2 & 0.9 \\
\hline 11 & $\mathrm{O} 1$ & $\mathrm{sp}^{2}$ oxygen & 95 & 1.85 & 1.0 \\
\hline 12 & $\mathrm{O} 2$ & $\mathrm{sp}^{3}$ oxygen & 132 & 1.5 & 0.8 \\
\hline 13 & $\mathrm{~F}$ & fluorine & 89 & 2.0 & 1.0 \\
\hline 14 & $\mathrm{P}$ & phosphorus & 9 & 2.5 & 0.5 \\
\hline 15 & $\mathrm{~S}$ & sulfur atom & 25 & 2.0 & 1.1 \\
\hline 16 & $\mathrm{Cl}$ & chlorine & 128 & 2.2 & 0.8 \\
\hline 17 & $\mathrm{Br}$ & bromine & 37 & 2.5 & 0.7 \\
\hline 18 & $\mathrm{~N} 2 \mathrm{C}$ & charged $\mathrm{sp}^{2}$ nitrogen atom & 24 & 2.8 & 0.6 \\
\hline 19 & $\mathrm{~N} 3 \mathrm{C}$ & charged $\mathrm{sp}^{3}$ nitrogen atom & 14 & 1.4 & 1.0 \\
\hline 20 & $\mathrm{OC}$ & charged oxygen atom & 8 & 1.3 & 1.9 \\
\hline 21 & $\mathrm{SC}$ & charged sulfur atom & 4 & 1.8 & 1.1 \\
\hline
\end{tabular}

molecules after minimization of molecular mechanics were further optimized using quantum mechanics with $\mathrm{HF} / 6-31 \mathrm{G}$ basis set, and then the HF/6-31G* electrostatic potential (ESP) charges of the small organic molecules were obtained using Gaussian-98. ${ }^{39}$ For proteins, their ESP charges were assigned using xleap, the graphics interface of AMBER 6.0 software package ${ }^{40}$ which assigned the predetermined RESP charge to each atom in protein. The charge we used here was the gasphase $\mathrm{HF} / 6-31 \mathrm{G}^{*}$ charge, which is larger than the condensedphase charge, but this error mimics the increased partial atomic charges that would be expected after solute polarization were the solute to be treated by a quantum mechanical self-consistent reaction field procedure. Carlson et al. were the first to point this out explicitly. ${ }^{40}$

Calibration of Atomic Effective Born Radii. The effective Born radii were calculated following a procedure recommended by Hawkins, Cramer, and Truhlar, in which the effective Born radii are estimated from a sum over atom pairs as described in eq $4 .^{18}$

$$
\begin{aligned}
R_{i}^{-1}=r_{i}^{-1}-\left(\frac{1}{2}\right) \sum_{j} \frac{1}{L_{i j}}-\frac{1}{U_{i j}}+\frac{r_{i j}}{4}\left(\frac{1}{U_{i j}^{2}}-\frac{1}{L_{i j}^{2}}\right)+ \\
\frac{1}{2 r_{i j}} \ln \frac{L_{i j}}{U_{i j}}+\frac{\rho_{j}^{2}}{2 r_{i j}}\left(\frac{1}{U_{i j}^{2}}-\frac{1}{L_{i j}^{2}}\right)
\end{aligned}
$$

where

$$
\begin{gathered}
L_{i j}=1, \quad \text { if } r_{i}>r_{i j}+\rho_{j} \\
L_{i j}=r_{i}, \quad \text { if } r_{i j}-\rho_{j}<r_{i}<r_{i j}+\rho_{j} \\
L_{i j}=r_{i j}-\rho_{j}, \quad \text { if } r_{i}<r_{i j}-\rho_{j} \\
U_{i j}=1, \quad \text { if } r_{i}>r_{i j}+\rho_{j} \\
U_{i j}=r_{i j}+\rho_{j}, \quad \text { if } r_{i}<r_{i j}+\rho_{j}
\end{gathered}
$$

In the above equations, $r_{i}$ and $r_{j}$ are the initial Born radii of atom $i$ and atom $j$, while and $\rho_{i}$ is the product of atom $i$ 's initial Born radii and screen parameters. The screen parameters were introduced by Hawkins et al. to correct for systematic errors introduced by the pairwise screening approximation.
Nonpolar Contribution of the Solvation Free Energy. As we had mentioned above, the nonpolar contribution solvation free energy is linear relative to the SASA. We calculated the surface using MSMS with a probe radius of $1.4 \AA .{ }^{41}$ The nonpolar contribution of solvation free energy is then estimated with a coefficient of $0.005 \mathrm{kcal} /\left(\mathrm{mol} \AA^{2}\right)$, which is the default value of the SANDER module of the AMBER 6.0 software package.

Parameters Fitting Procedure. Now we have all the inputs required by a GB/SA solvation model. Given a set of parameters, we can predict the solvation free energy of each molecule in the training set, and given the experimental solvation free energy, we can estimate its mean unsigned error.

Here, the fitting procedure was based on GA, which was under development in our laboratory. ${ }^{30-32}$ GA can effectively deal with the multiple-dimension problem, no matter whether those variables are highly coupled or not, which makes it an ideal optimization method for the problem of parametrization. ${ }^{33,42,43}$ The brief fitting process based on GA is in four steps: creation of the initial population, selection operation, crossover operation, and mutation operation. According to the GA, an individual should be represented as a linear string, which plays the role of the DNA for the individual, so the parameters for all atom types were treated as a string. The initial population was generated by randomly generating the initial parameters. Then these individuals were scored according to their fitness. In the parametrization of GA, the sum of mean unsigned errors of the training set was used as the score function. After some cycles of the selection, crossover, and mutation operations, the model with the highest fitness score was obtained. The GA optimizations were terminated if the total mean unsigned error did not change after a certain number of iterative cycles; for example, after 100, the optimization would end. More detailed description of the fitting process based on GA can be found in our previous work. $33,42,43$

The fitting procedure was taken in two stages. In the first stage, the parameters of 17 normal atom types were determined by fitting the experimental solvation free energy with the calculated solvation free energies of the 328 neutral using a genetic algorithm. In the second stage, the derived parameter in the first stage was fixed and the parameters of four ion atom types were determined by fitting the experimental solvation free 
energies with the calculated solvation free energies of the 30 charged molecules using a systemic search method.

PB/SA Solvation Model. As we had mentioned above, PB/ SA solvation model is a widely accepted solvation model, so we used the model to predict the solvation free energy of test set molecules, and 15 proteins for a reference.

In PB/SA model, the electrostatic contribution to the solvation free energy was calculated by taking the difference between the total energy of the system obtained with $\epsilon$ int $=2 ; \epsilon$ ext $=1$ and $\epsilon$ int $=2 ; \epsilon$ ext $=78.5$. It requires an input of atomic coordinate, partial charge, and van der Waals radii. In our calculations, we used the same partial charge and coordinate as in the GB calculation. For the van der Waals radii, we used the default value supplied by the DelphiII software package. ${ }^{19}$ The radii of atoms were taken from the PARSE parameter set. ${ }^{44}$ It should be noted that the PARSE parameter set does not provide the van der Waals radii of halogen, so we used the van der Waals radii in the AMBER force field instead, which is $1.75 \AA$ for the fluorine atom, $1.95 \AA$ for the chlorine atom, and $2.22 \AA$ for the bromine atom.

The calculations based on PB equations were performed using the DelphiII software package. In the prediction of solvation free energies of test set molecules, the resolution employed was 4 grids/ $\AA$. For proteins, the resolution was 1.8 grids $/ \AA$. The calculations of SASA were performed using the MSMS program..$^{41}$

Jayaram's GB/SA Model. Jayaram's generalized Born model was employed to predict the solvation free energy of all the reference systems. ${ }^{21}$ The setting is the same as that in our GB calculations.

It should be noted that many molecules in the training set and the test set contain halogen atoms, but they do not have corresponding GB parameters in Jayaram's parameter set, so for these atoms, the initial Born radii were set to van der Waals radii in the AMBER force field and the screen parameter was set to 1.0. The calculations based on Jayaram's solvation model were performed using SANDER in AMBER6.0 with the control parameter GBPARM set to 1 .

\section{Results and Discussion}

Atom Typing Rules. As we mentioned above, for each atom two parameters should be determined: the initial Born radii and the screen parameter, so the determination of the parameter of every atom is impossible. One strategy is to classify atoms into different categories according to its chemical environment. Atoms in the same category have the same parameters. Each category is called an atom type, and their definitions are called the atom typing rule. The atom typing rule is the key of the parametrization work, and it should meet some demands. First, it should be fully contained, which means that every atom in the concerned system can be classified into one atom type; second, each atom type should be exclusive, which means there should not be an atom that can be classified into two or more categories; third, the number of atom types should be as small as possible to avoid the overfitting problem.

In the work of Jayaram et al., ${ }^{24}$ the authors employed relative simple atom typing rules, in which atoms are classified into six types according to their element. They chose this typing rule partly because of their relative small training set (32 molecules). Their parameters would be efficient in predicting the solvation free energy of proteins and nuclear acids, because atom types in these systems are very limited, but it may not behave well in predicting the solvation free energies of organic molecules because the chemical environments in organic molecules are
TABLE 2: Neutral Organic Molecules in the Training Set with Deviations Larger than $2.0 \mathrm{kcal} / \mathrm{mol}$

\begin{tabular}{rlrrr}
\hline no. & \multicolumn{1}{c}{ molecular name } & $\Delta G_{\text {exp }}$ & $\Delta G_{\text {calc }}$ & residue \\
\hline 65 & tetrafluoromethane & 3.16 & 0.33 & -2.83 \\
66 & hexafluoroethane & 3.94 & 1.07 & -2.87 \\
67 & octafluoropropane & 4.28 & 2.01 & -2.27 \\
68 & fluorobenzene & 0.78 & -1.71 & -2.49 \\
70 & chlorofluoromethane & 0.77 & -1.36 & -2.13 \\
90 & $1,1,2,2$-tetrachloroethane & -2.36 & -0.08 & 2.28 \\
225 & methyl formate & -2.78 & -5.91 & -3.13 \\
226 & ethyl formate & -2.65 & -5.29 & -2.64 \\
227 & propyl formate & -2.48 & -5.21 & -2.73 \\
251 & ethyl heptanoate & -4.60 & -7.87 & -3.27 \\
252 & methyl octanoate & -4.61 & -6.72 & -2.11 \\
253 & methyl benzoate & -4.50 & -7.55 & -3.05 \\
254 & butylamine & -4.38 & -6.65 & -2.27 \\
255 & pentylamine & -4.09 & -6.67 & -2.58 \\
256 & hexylamine & -4.04 & -6.82 & -2.78 \\
264 & $N, N$-dimethylpiperazine & -7.58 & -2.04 & 5.55 \\
265 & $N$-methylpiperazine & -7.77 & -5.44 & 2.33 \\
266 & 1,1-dimethyl-3-phenyl urea & -11.87 & -9.00 & 2.87 \\
268 & ethylenediamine & -9.75 & -18.23 & -8.48 \\
271 & $N$-methylmorpholine & -6.34 & -3.59 & 2.75 \\
272 & $N$-methylpyrrolidine & -3.97 & -0.80 & 3.17 \\
273 & $N$-methylpiperidine & -3.89 & -0.78 & 3.11 \\
291 & 9 -methyladenine & -13.60 & -20.19 & -6.59 \\
307 & $N$-methylformamide & -10.00 & -7.94 & 2.06 \\
309 & $E-N$-methylacetamide & -10.00 & -7.75 & 2.25 \\
310 & $Z$ - $N$-methylacetamide & -10.00 & -7.14 & 2.86 \\
328 & dimethyl 3-methyl-4-thio- & -6.92 & -3.85 & 3.07 \\
& methoxyphenyl thiophosphate & & & \\
329 & ethyl 4-cyanophenyl & -5.10 & -7.91 & -2.81 \\
& phenylthiophosphonate & & & \\
& & & &
\end{tabular}

much more complicated than those in proteins. Therefore, Jayaram's parameters may not be suitable for studying the solvation contribution to the interaction free energy between enzyme and inhibitor, which is of great importance in drug design.

To predict the solvation free energy of organic molecules, more complicated atom typing rules must be employed. Here we employed an atom typing rule containing 21 atom types, and four of them are specially designed for representing ions. Their definitions and number of appearance in the training set are listed in Table 1. To avoid the overfitting problem, we employed a much bigger training set made up of 358 molecules, in which 328 are neutral and 30 are charged.

We took this typing rule from the one SANDER used in the calculation of molecular SASA. As we know SANDER employed a method named LCPO to calculate molecular SASA. In LCPO, atoms were classified into 21 types according to their element, hybridization, and number of hydrogen atoms linked to it; our typing rule is much like theirs except that we neglect the number of hydrogen atoms which will enlarge our type set and consequently cause the overfitting problem.

Solvation Free Energy of Training Set and Test Set. The derived parameters are listed in Table 1 . If we do not consider the four charged atom types, the GB/SA model based on the new parameters yielded fairly satisfactory results, $n=328$, $r=0.911, s=1.241, F=1590.841$. From the predictions to the molecules in the training set, there are two compounds (compounds 268 and 291 in Table A, Supporting Information) with deviations larger than $6.0 \mathrm{kcal} / \mathrm{mol}$. If we eliminate these two compounds as outliers, the correlation between the experimental solvation free energies and the calculated values was improved obviously $(n=326, r=0.924, s=1.115, F=$ 1911.039). For the 328 neutral molecules in the training set, this new set of parameters gives a mean unsigned error of 0.85 $\mathrm{kcal} / \mathrm{mol}$ in predicting the solvation free energy. For the charged 
TABLE 3: Solvation Free Energy of Molecules in the Test Set

\begin{tabular}{|c|c|c|c|c|c|}
\hline no. & name & $\Delta G_{\exp }$ & our GB/SA model & PB/SA model & Jayaram's GB/SA model \\
\hline A1 & $n$-pentane & 2.33 & 1.18 & 1.13 & 1.67 \\
\hline A2 & $n$-heptane & 2.62 & 1.32 & 1.29 & 1.96 \\
\hline A3 & 4-methyl-1-pentene & 1.91 & 1.15 & -1.01 & -0.19 \\
\hline A4 & 1,4-pentadiene & 0.94 & 0.98 & -2.46 & -1.29 \\
\hline A5 & butenyne & 0.04 & -0.07 & -4.01 & -3.13 \\
\hline A6 & butylbenzene & -0.40 & -1.30 & -2.16 & 0.67 \\
\hline A7 & 1,1-difluoroethane & -0.11 & -1.34 & -2.92 & -0.59 \\
\hline A8 & dichlorodifluoromethane & 1.69 & 1.15 & 1.14 & 1.68 \\
\hline A9 & 1-bromo-1,2,2,2-tetrafluoroethane & 0.52 & -1.30 & -0.86 & 2.88 \\
\hline A10 & 1,1,1,2-tetrachloroethane & -1.15 & -0.36 & -1.53 & 1.39 \\
\hline A11 & 1,1-dichlorobutane & -0.70 & -0.51 & -1.47 & 0.84 \\
\hline A12 & chlorobenzene & -1.01 & -1.60 & -2.17 & 0.36 \\
\hline A13 & 1-chloro-2-bromoethane & -1.95 & -0.82 & -2.81 & 0.28 \\
\hline A14 & 1-bromo-2-methylpropane & -0.03 & -0.48 & -1.61 & 0.17 \\
\hline A15 & $o$-bromocumene & -0.85 & -1.31 & -2.03 & 0.51 \\
\hline A16 & 1-butanol & -4.72 & -5.24 & -4.12 & -1.97 \\
\hline A17 & 2-methyl-1-pentanol & -3.93 & -4.62 & -3.79 & -1.51 \\
\hline A18 & 1-heptanol & -4.25 & -4.94 & -3.79 & -1.42 \\
\hline A19 & 3 -cresol & -5.49 & -5.86 & -7.02 & -3.04 \\
\hline $\mathrm{A} 20$ & ethyl propyl ether & -1.81 & -1.23 & -0.89 & 1.25 \\
\hline $\mathrm{A} 21$ & 1,2-diethoxyethane & -3.53 & -2.43 & -2.16 & 1.46 \\
\hline A22 & pentanal & -3.03 & -2.89 & -4.26 & -3.16 \\
\hline A23 & $m$-hydroxybenzaldehyde & -9.51 & -8.80 & -10.22 & -5.51 \\
\hline A24 & cyclopentanone & -4.68 & -3.32 & -4.76 & -2.98 \\
\hline $\mathrm{A} 25$ & propionic acid & -6.46 & -6.36 & -8.11 & -4.80 \\
\hline A26 & isobutyl formate & -2.22 & -3.13 & -5.24 & -1.68 \\
\hline A27 & methyl propionate & -2.97 & -3.01 & -4.93 & -1.54 \\
\hline A28 & methyl hexanonate & -2.48 & -2.57 & -4.58 & -0.84 \\
\hline A29 & dimethylamine & -4.28 & -4.99 & -1.98 & -0.36 \\
\hline A30 & aniline & -5.49 & -7.42 & -7.69 & -2.31 \\
\hline A31 & pyrrolidine & -5.47 & -2.88 & -1.24 & 0.84 \\
\hline A32 & 2,4-dimethylpyridine & -4.85 & -4.39 & -4.33 & -1.44 \\
\hline A33 & 2-ethyl-3-methoxypyrazine & -4.39 & -4.20 & -3.48 & -0.10 \\
\hline A34 & $N^{\prime}, N$-dimethyl formamide & -4.90 & -5.38 & -6.81 & -4.45 \\
\hline A35 & thioanisole & -2.73 & -2.09 & -3.20 & -0.51 \\
\hline \multirow[t]{2}{*}{ A36 } & tripropyl phosphate & -6.10 & -6.47 & -8.58 & -3.14 \\
\hline & mean unsigned error & 0.00 & 0.73 & 0.73 & 1.56 \\
\hline A37 & $\left(\right.$ cyclo $\left.-\mathrm{C}_{6} \mathrm{H}_{11}\right) \mathrm{NH}_{3}{ }^{+}$ & -62.00 & -56.91 & -66.08 & -42.55 \\
\hline A38 & $\left(\mathrm{CH}_{3}\right)\left(\mathrm{C}_{6} \mathrm{H}_{5}\right) \mathrm{NH}_{2}^{+}$ & -56.50 & -56.16 & -62.38 & -40.48 \\
\hline A39 & $\mathrm{C}_{5} \mathrm{H}_{5} \mathrm{NH}^{+\mathrm{c}}$ & -53.50 & -59.05 & -64.08 & -46.55 \\
\hline $\mathrm{A} 40$ & $\left(\mathrm{CH}_{3}\right)_{2}\left(n-\mathrm{C}_{3} \mathrm{H}_{7}\right) \mathrm{NH}^{+}$ & -59.00 & -51.63 & -58.67 & -35.84 \\
\hline A41 & $\left(n-\mathrm{C}_{3} \mathrm{H}_{7}\right) \mathrm{H}_{5} \mathrm{~N}_{3} \mathrm{C}^{+}$ & -65.50 & -61.15 & -63.21 & -34.23 \\
\hline A42 & $\left(i-\mathrm{C}_{3} \mathrm{H}_{7}\right) \mathrm{H}_{5} \mathrm{~N}_{3} \mathrm{C}^{+}$ & -63.00 & -62.00 & -63.64 & -30.95 \\
\hline A43 & $\mathrm{C}_{6} \mathrm{H}_{5} \mathrm{CO}_{2}^{-}$ & -73.50 & -68.05 & -68.42 & -62.06 \\
\hline \multirow[t]{2}{*}{ A44 } & $\left(n-\mathrm{C}_{3} \mathrm{H}_{7}\right) \mathrm{S}^{-}$ & -79.00 & -78.88 & -78.81 & -71.71 \\
\hline & mean unsigned error & 0.00 & 3.65 & 3.65 & 3.63 \\
\hline
\end{tabular}

molecules in the training set, the GB/SA calculations give much worse prediction than the neutral molecules. For the 30 charged molecules in the training set, the new parameters produced a mean unsigned error of $4.71 \mathrm{kcal} / \mathrm{mol}$. The experimental and calculated solvation free energies using the new parameters are summarized in Tables A and B in Supporting Information. The GB/SA model based on our new parameters predicts well for most of the 328 neutral compounds in training set, but as listed in Table A and B, 28 compounds showed deviations greater than $2.0 \mathrm{kcal} / \mathrm{mol}$. We think that these deviations can be explained by two reasons. The first may be suggested by the principle difference between the experimental solvation free energies and the calculated values of GB/SA. In our fitting process, the experimental solvation free energies were treated as the standard values. But it should be noted that the experimental value of a solute is not induced by a single molecule, but by a group of solute molecules in solvent. The solute molecules may produce intermolecular or intramolecular group-group interactions, for example, the intermolecular hydrophobic interactions and intra- or intermolecular hydrogen bonds. Jayaram et al. used the solvation free energy predicted by the PB/SA model as the standard value; it can be accurate in dealing with protein systems, but it is unacceptable in dealing with small organic molecule systems, as we illustrated in Table 3. The PB/SA model cannot give accurate solvation free energies of small organic molecules, so we think that using the experimental solvation free energies in parametrization is more reasonable than the calculated solvation free energies from $\mathrm{PB} /$ SA calculations. Second, in our work, we may not define the best atom typing rules. In principle, if we define enough atom types and can obtain the parameters for them, the electrostatic contribution may be well estimated. But unfortunately, the chemical environments in organic molecules are so complicated that it is very difficult for us to define unlimited atom types to differentiate all chemical environments. It could explain the stunningly large radius of type N2C (2.8 $)$, but we have very little occurrence of this type of atom ( 24 times), so the radius of this type is not reliable. It could be overcome by adding a new experimental value to the training set. Moreover, the data set with experimental solvation free energies is limited. The data do not allow us to define so many atom types, otherwise overfitting cannot be avoided.

The derived parameters were then applied to predict the solvation free energy of the test set molecules for validation. 

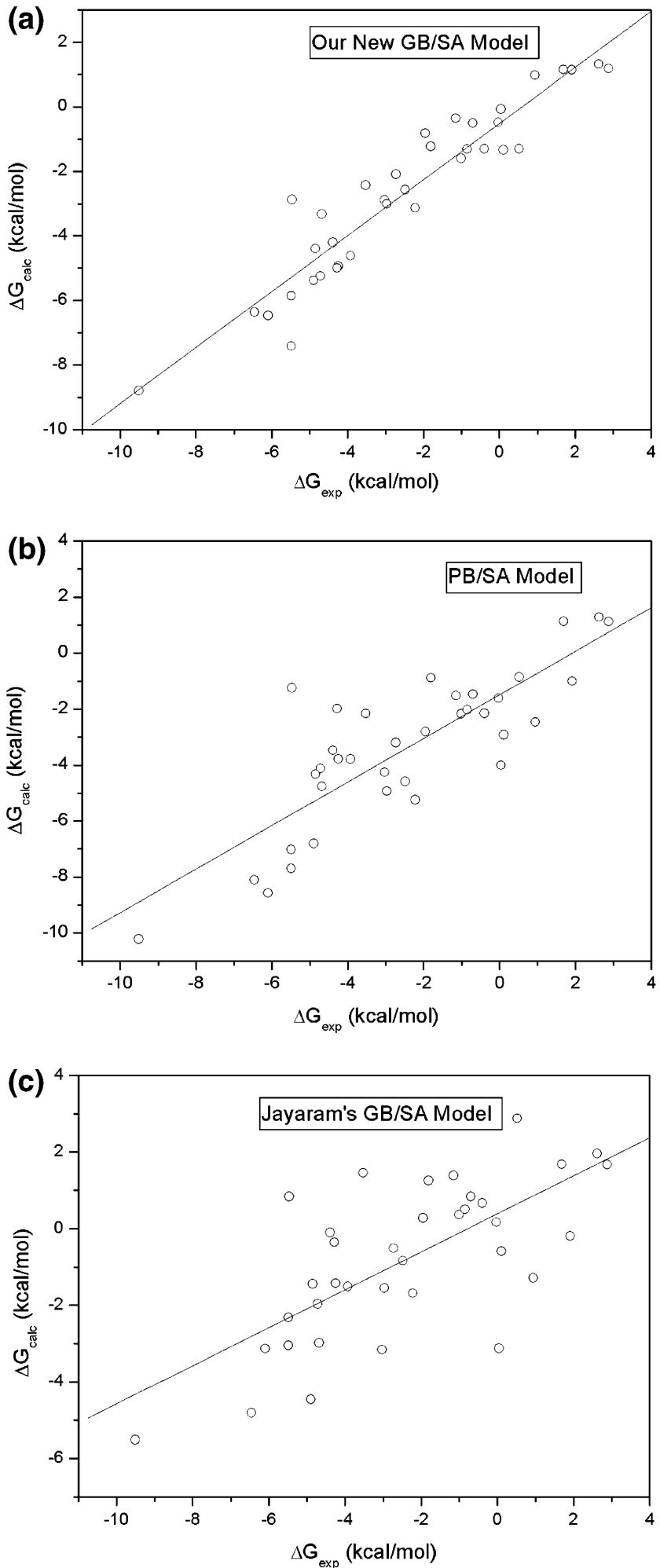

Figure 1. (a) Experiment solvation free energy vs predicted value using our new parameters. (b) Experiment solvation free energy vs predicted value using our new parameters. (c) Experiment solvation free energy vs predicted value using Jayram's GB/SA model.

The experimental solvation free energy and the predicted value of the test set are listed in Table 3 . For the 36 neutral molecules in the test set, the obtained mean unsigned error is $0.73 \mathrm{kcal} /$ $\mathrm{mol}$, and for the left 8 charged molecules this value is 3.65 $\mathrm{kcal} / \mathrm{mol}$. Figure 1a shows the linear correlation between the experimental values and the calculated values using GB/SA based on our new parameters for the neutral molecules in the
TABLE 4: Solvation Free Energy of Proteins

\begin{tabular}{cccc}
\hline PDB entry & PB/SA & Jayaram's GB/SA & our GB/SA \\
\hline 1ahh & -4013 & -1170 & -4047 \\
1bbh & -2221 & -2966 & -3387 \\
1bbs & -3769 & -3200 & -3745 \\
1c7c & -5260 & -1615 & -4057 \\
1ctf & -1087 & -1018 & -1029 \\
1dyv & -1586 & -1648 & -2100 \\
1eol & -3100 & -2269 & -3320 \\
1fkh & -1074 & -809 & -983 \\
1g54 & -2283 & -1731 & -2279 \\
1gky & -2268 & -2047 & -2286 \\
1htr & -4517 & -3666 & -4084 \\
1mpp & -6090 & -6001 & -6056 \\
1prn & -7788 & -7930 & -7503 \\
1ypa & -681 & -625 & -697 \\
2alp & -1468 & -742 & -1537
\end{tabular}

test set, which has a correlation coefficient of 0.95 and a standard deviation of 0.86 . The good prediction for the test set indicates that the obtained parameters are reliable.

In a comparative fashion, the PB/SA model and Jayaram's GB/SA model had also been employed to predict the solvation free energy of the test set. For the charged molecules in the test set, the performance of our GB/SA model, the PB/SA model, and Jayaram's GB/SA is similar. But for the neutral molecules in the test set, the performance of these three solvation models shows obvious differences. Figure $1 \mathrm{~b}$ shows the linear correlation between the experimental values and the calculated values using $\mathrm{PB} / \mathrm{SA}$ for the neutral molecules in the test set, which has a correlation coefficient of 0.82 and a standard deviation of 1.57. For the neutral molecules in Table 3, the mean unsigned error between the experimental values and the calculated values using $\mathrm{PB} / \mathrm{SA}$ is $1.55 \mathrm{kcal} / \mathrm{mol}$. It is obvious that the predictive ability of our GB/SA model is obviously better than that of the PB/SA model. Figure 1c shows the linear correlation between the experimental values and the calculated values using Jayram's GB/SA model for the neutral molecules in the test set, which has a correlation coefficient of 0.70 and a standard deviation of 1.48. Meanwhile, the unsigned mean error is $2.18 \mathrm{kcal} / \mathrm{mol}$, which means that Jayaram's GB/SA model does not have good predictive power for small organic molecules. From the calculated results in Table 3, we also find that the PB/SA model and Jayaram's GB/SA model failed in predicting the solvation free energy of molecules containing halogen. That is because the two models do not contain parameters for halogen atoms. We also see that Jayaram's GB/ SA model fails to predict the solvation free energy of molecules containing pyrrole or pyridine functional groups. This may be because they did not include this kind of molecule in their training set. The fact that the PB/SA model behaved much better than Jayaram's model implies that the PB/SA model is a more reliable model than the GB/SA model. Though our new model behaved better than the PB/SA model, our model employed 34 parameters, while the PB/SA employed only nine parameters. The only superiority of the GB/SA model to the PB/SA model is that the GB/SA model is much faster than the PB/SA model. To perform $\mathrm{PB}$ calculations on the 36 molecules in test set, it took a PIV1.4GHz processor $33 \mathrm{~s}$. For the GB model, the cost time is only $3 \mathrm{~s}$.

Solvation Free Energy of Proteins. The parameters for GB/ SA are derived based on a set of small molecules. Certainly, the functional groups of protein can also be found in these small organic molecules, so we believe that the parameters can be extended to proteins. The calculated results using our GB/SA model and the PB/SA model for the 15 proteins in the test set are shown in Table 4. Figure $2 \mathrm{a}$ shows that the plot of 

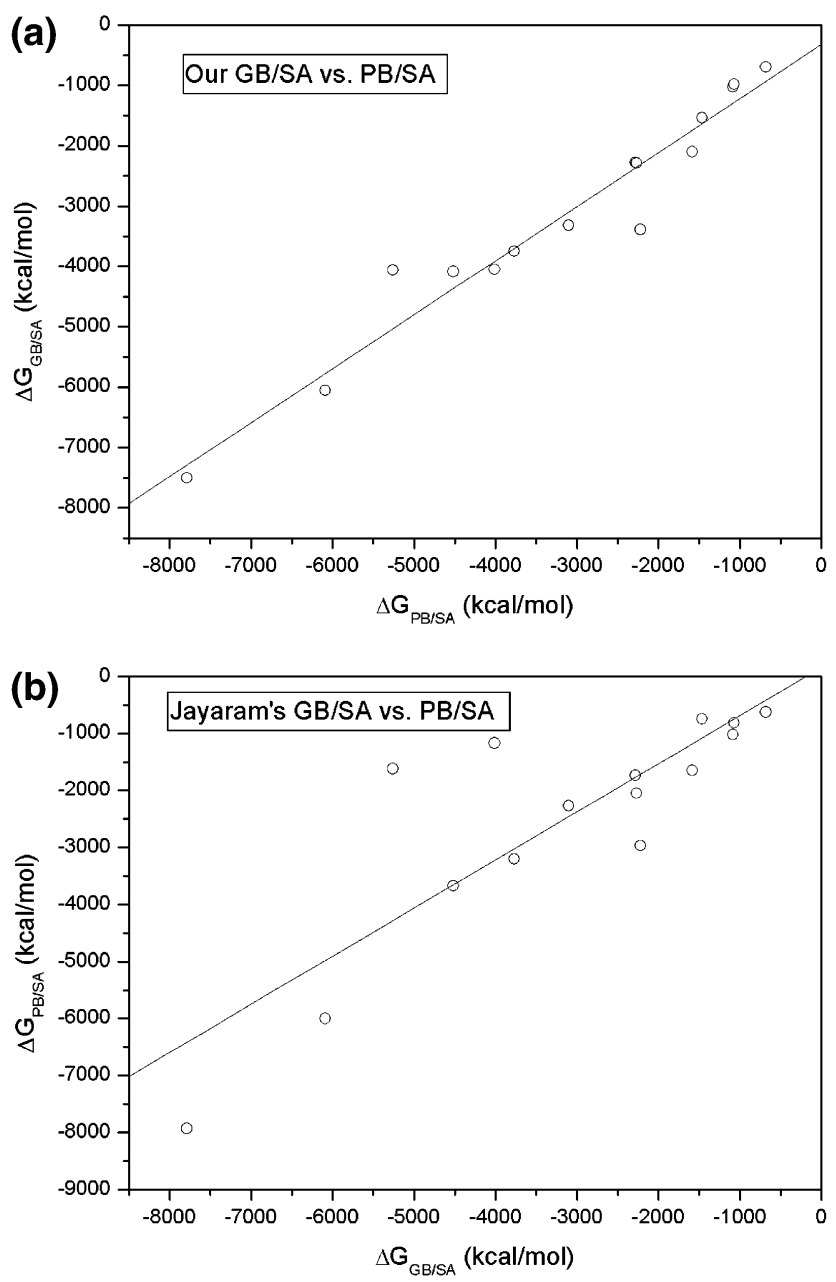

Figure 2. (a) The predicted solvation free energy of proteins using our GB/SA model vs PB/SA model. (b) The predicted solvation free energy of proteins using Jayaram's GB/SA vs PB/SA model.

predictions using $\mathrm{PB} / \mathrm{SA}$ versus predictions using our GB/SA model. The good linear correlation $(r=0.97)$ indicates that the solvation abilities of these 15 proteins can be well ranked by the predictions using our GB/SA model. From the absolute values, the predictions using $\mathrm{PB} / \mathrm{SA}$ are in good agreement with those using our GB/SA model besides $1 \mathrm{bbh}$ and $1 \mathrm{bbs}$.

Here, the predictive ability of Jayaram's GB/SA model was also investigated. The predicted values are shown in Table 4. The correlation between the predictions using Jayaram's GB/ SA model and those using PB/SA is shown in Figure $2 \mathrm{~b}$. The predicted values using these two models show obvious linear correlation $(r=0.85)$. But the linear correlation is obviously worse than that shown in Figure 2b. Moreover, the data in Table 4 indicate that the unsigned mean error between the predicted values using $\mathrm{PB} / \mathrm{SA}$ and those using Jayaram's GB/SA model is $778 \mathrm{kcal} / \mathrm{mol}$, which is much larger than the unsigned mean error $(278 \mathrm{kcal} / \mathrm{mol})$ between the predicted values using PB/ SA and those using our GB/SA model. This fact implies that for proteins the predictive power of our GB/SA model is much better than that of Jayaram's GB/SA model.

Further Development and Applications of the GB/SA Model. Due to the simplicity and efficiency of the GB/SA model, it may be widely used in many fields. But further application of this method is also significantly restricted by its predictive power. If we want to improve the predictive ability of the GB/SA model, we should provide more elaborate atom typing rules and corresponding parameters. Although the predictions of the GB/SA model using our new parameters have been improved a lot, we also believe that the atom typing rules used here should not be the optimum. In our further work, we will attempt to give more rational definitions for these atoms in a complicated chemical environment. Certainly, the number of atom types is strongly limited by the available experimental data. The predictive ability of the charge-independent model may be improved by choosing a balanced training set that represents as many chemical functionalities as possible.

The further potential applications of GB/SA should be promising. First, the SASA model has potential applications in molecular dynamics, conformational analysis, and protein folding. For example, the special program named $p d b \_t y p i n g$ was developed to help current AMBER users apply our new parameters to their MD simulations. More detailed descriptions of the program $p d b \_t y p i n g$ can be found in Supporting Information. In our previous work, GA was used to sample the conformational spaces and thoroughly search the global conformations of peptides. ${ }^{41}$ But in our program, only the potentials of the peptides were considered. In future work, we will apply this model to calculate the solvation free energy in protein folding or the installation of side chains. We expect that the consideration of the solvation free energy will improve the performance of our method. Second, we will apply this model to calculate the relative binding free energy for a set of protein/ ligand complexes and incorporate this model into our docking program. In our group, we have developed different score functions for the following two stages of conformation searching. In the first stage, surface complementarity is considered, while in the second stage only energetic complementarity is considered. In the current release of our SFDOCK program, only the van der Waals and electrostatic interactions were used to estimate the energetic complementarity. Soon, the GB/SA model will be incorporated into our program.

\section{Conclusion}

We derived a set of parameters for the GB/SA model consistent with the AMBER force field. We employed a much larger training set (358 molecules) in the parameter's derivation procedure than Jayaram et al. did in deriving their parameters. In the current work, we employed atom typing rules containing 21 atom types, of which four are specially designed for ions. The definition of atom types was based on the SMARTS string. Predictions using the solvation model based on the 358-molecule set give an average unsigned error of $0.85 \mathrm{kcal} / \mathrm{mol}$ for the neutral molecules and $4.71 \mathrm{kcal} / \mathrm{mol}$ for the ions.

We applied the parameters developed in this paper to calculate the solvation free energies for 44 small organic molecules. The calculated results using our new parameters are consistent with those from experiments. Comparison of the results from our GB/SA model, PB/SA model, and Jayaram's GB/SA model shows that the calculations with our GB/SA model are obviously better than those with the other two solvation models. We have also applied our model to predict the solvation free energies for 15 proteins. For the 15 proteins randomly selected from the Brookhaven PDB database, the solvation free energies predicted by the SASA model bear high linear correlations $(r=0.97)$ with those predicted by the PB/SA model, which were much better than those given by Jayaram's GB/SA model.

Supporting Information Available: Experimental and calculated solvation free energy values for molecules of the training set (Table A) and experimental and calculated free 
energy values for molecules in the test set (Table B). This material is available free of charge via the Internet at http:// pubs.acs.org. The special program $p d b \_t y p i n g$ was developed to help AMBER 6.0 users apply our parameters to their MD simulations. pdb_typing reads the coordinates with PDB format and writes a radii file that contains the radii and screen parameter of each atom. This program needs two input files: gbparm.dat and atomtyp.txt. File gbparm.dat contains the derived GB/SA parameters of our work, and atomtyp.txt contains the atom typing definitions represented in SMARTS language. AMBER users should do a minor revision to SANDER's source code, which is under the directory \$AMBERHOME/src/sander/. The revision is adding one line "read $\left(18,{ }^{*}\right)(x(\mathrm{~L} 96-1+\mathrm{i}), \mathrm{i}=1$, natom)" to the file mdread.f after line 637, and then recompiling it. (You can also download the revised file from our web site.) The PDB format file can be generated using the SAVEPDB command available in XLEAP module of AMBER6.0. The generated radii file can be used by SANDER in MD simulations and optimizations by specifying -radii <filename> in the command line and setting the parameter READRAD to 1 in SANDER's control parameter file. The program $p d b \_t y p i n g$ and the corresponding parameter files can be obtained from us upon request.

\section{References and Notes} 1937.

(1) Rossky, P. J.; Karplus, M. J. Am. Chem. Soc. 1979, 101, 1913-

(2) Jorgensen, W. L.; Chandrasekhar, J.; Madura, J. D.; Impey, R. W.; Klein, M. L. J. Chem. Phys. 1983, 79, 926-935. 3054 .

(3) Jorgensen, W. L.; Ravimohan, C. J. J. Chem. Phys. 1985, 83, 3050-

(4) Eisenberg, D.; McLachlan, A. D. Nature 1986, 319, 199-203.

(5) Ooi. T.; Oobatake, M.; Nemethy, G.; Scheraga, H. A. Proc. Natl. Acad. Sci. U.S.A. 1987, 84, 3086-3090.

(6) Kang, Y. K.; Nemethy, G.; Scheraga, H. A. J. Phys. Chem. 1987, 91, 4105-4109/4109-4118.

(7) Kang, Y. K.; Nemethy, G.; Scheraga, H. A. J. Phys. Chem. 1987, 91, 4118-4122.

(8) Gilson, M.; Honig, B. Proteins 1988, 4, 7-18.

(9) Still, W. C.; Tempczyk, A.; Hawley, R. C.; Hendrickson, T. J. J. Am. Chem. Soc. 1990, 112, 6127-6133. 8311 .

(10) Cramer, C. J.; Truhlar, D. G.J. Am. Chem. Soc. 1991, 113, 8305-

(11) Hermann, R. B. J. Phys. Chem. 1972, 76, 2754-2759.

(12) Amidon, G. L.; Yalkowsky, S. H.; Anik, S. T.; Valvani. S. C. J. Phys. Chem. 1975, 79, 2239-2246.

(13) Floris, F.; Tomasi, J. J. Comput. Chem. 1989, 10, 616-627.

(14) Hou, T. J.; Qiao, X. B.; Zhang, W.; Xu, X. J. J. Phys. Chem. B 2002, 106, 11295-11304.

(15) Hou, T. J.; Xu, X. J. Acta Phys. Chim. Sin. 2002, 18, 1052-1056.

(16) Jayaram, B. J. Phys. Chem. 1994, 98, 5773-5777.

(17) Nicholls, A.; Honig, B. J. Comput. Chem. 1991, 12, 435-445.

(18) Gilson, M.; Sharp, K. A.; Honig, B. J. Comput. Chem. 1988, 9 , 327-335.

(19) Honig, B.; Nicholls, A. Science 1995, 268, 1144-1149

(20) Hawkins, G. D.; Cramer, C. J.; Truhlar, D. G. Chem. Phys. Lett. 1995, 246, 122-129.

(21) Cramer, C. J.; Truhlar, D. G. Chem. Rev. 1999, 99, 2161-2200.
152

(22) Bashford, D.; Case, D. A. Annu. Rev. Phys. Chem. 2000, 51, 129-

(23) Orozco, M.; Luque, F. J. Chem. Rev. 2000, 100, 4187-4225.

(24) Jayaram, B.; Sprous, D.; Beveridge, D. L. J. Phys. Chem. B 1998, 102, 9571-9576.

(25) Cheng, A.; Best, S. A.; Merz, J. K. M.; Reynolds, C. H. J. Mol. Graph. Mod. 2000, 18, 273-282.

(26) Zhu, J.; Shi Y. Y.; Liu, H. Y. J. Phys. Chem. B 2002, 106, 48444853.

(27) Judson, R. S.; Jaeger, E. P.; Treasurywala, A. M.; Peterson, M. L. J. Comput. Chem. 1993, 14, 1407-1414.

(28) Oshiro, C. M.; Kuntz, I. D.; Dixon, J. S. J. Comput. Aid. Mol. Des. $1995,9,113-130$

(29) Jones, G.; Willett, P.; Glen, R. C. J. Mol. Biol. 1995, 245, 43-53.

(30) Hou, T. J.; Wang, J. M.; Chen, L. R.; Xu, X. J. Protein Eng. 1999 $12,639-647$

(31) Kubinyi, H. Quantum Struct.-Act. Relat. 1994, 13, 285-294.

(32) Rogers, D.; Hopfinger, A. J. J. Chem. Inf. Comput. Sci. 1994, 34, 854-866.

(33) Hou, T. J.; Wang, J. M.; Liao, N.; Xu, X. J. J. Chem. Inf. Comput. Sci. 1999, 39, 775-781.

(34) Hawkins, G. D.; Cramer, C. J.; Truhlar, D. G. J. Phys. Chem. 1996, 100 (51), 19824-19839.

(35) Cerius2 User Guide; MSI: San Diego, USA, 1998.

(36) Halgren, T. A. J. Comput. Chem. 1996, 17, 490-519.

(37) InsightII User Guide; MSI: San Deigo, USA, 1998.

(38) James, C. A.; Weininger, D.; Delany, J. Daylight Theory Manual Daylight 4.62; Daylight Chemical Information Systems Inc.: Los Altos, 2001.

(39) Frisch, M. J.; Trucks, G. W.; Schlegel, H. B.; Scuseria, G. E.; Bobb, M. A.; Cheeseman, J. R.; Zakrzewski, V. G.; Montgomery, J. A.; Stratmann, R. E.; Burant, J. C.; Dapprich, S.; Millam, J. M.; Daniels, A. D.; Kudin, K. N.; Strain, M. C.; Farkas, O.; Tomasi, J.; Barone, V.; Cossi, M.; Cammi, R.; Mennucci, B.; Pomelli, C.; Adamo, C.; Clifford, S.; Ochterski, J.; Petersson, G. A.; Ayala, P. Y.; Cui, Q.; Morokuma, K.; Malick, D. K.; Rabuck, A. D.; Raghavachari, K.; Foresman, J. B.; Cioslowski, J.; Ortiz, J. V.; Stefanov, B. B.; Liu, G.; Liashenko, A.; Piskorz, P.; Komaromi, I.; Gomperts, R.; Martin, R. L.; Fox, D. J.; Keith, T.; Al-Laham, A.; Peng, C. Y.; Nanayakkara, A.; Gonzalez, C.; Challacombe, M.; Gill, P. M. W.; Johnson, B. G.; Chem, W.; Wong, M. W.; Andres, J. L. Head-Gordon, M.; Replogle, E. S.; Pople, J. A. Gaussian 98; Gaussian, Inc.: Pittsburgh, PA, 1998.

(40) Case, D. A.; Pearlman, D. A.; Caldwell, J. W.; Cheatham, T. E., III; Ross, W. S.; Simmerling, C. L.; Darden, T. A.; Merz, K. M.; Stanton, R. V.; Cheng, A. L.; Vincent, J. J.; Crowley, M.; Tsui, V.; Radmer, R. J.; Duan, Y.; Pitera, J.; Massova, I.; Seibel, G. L.; Singh, U. C.; Weiner, P. K.; Kollman, P. A. AMBER 6; University of California: San Francisco, 1999.

(41) Sanner, M. F.; Olson, A. J.; Spehner, J. Biopolymers 1996, 38, $305-320$.

(42) Hou, T. J.; Wang, J. M.; Li, Y. Y.; Xu, X. J. Chin. Chem. Lett. 1998, 9, 651-654.

(43) Hou, T. J.; Wang, J. M.; Xu, X. J. Chemometr. Intell. Lab. 1999, $45,303-310$

(44) Sitkoff, D.; Sharp, K. A.; Honig, B. Accurate Calculation of Hydration Free Energies Using Macroscopic Solvent Models. J. Phys. Chem. 1994, 98, 1978-1988.

(45) Wang, J. M.; Hou, T. J.; Chen, L. R.; Xu, X. J. Chemometr. Intell. Lab. 1999, 45, 347-351.

(46) Hou, T. J.; Wang, J. M.; Chen, L. R.; Xu, X. J. Protein Eng. 1999, $12,639-647$.

(47) Hou, T. J.; Wang, J. M.; Xu, X. J. Chin. Chem. Lett. 1999, 10, 615-618.

(48) Hou, T. J.; Xu, X. J. J. Mol. Graph. Model. 2001, 19, 455-465.

(49) Weiser, J.; Peter S. S.; Still, W. C. J. Comput. Chem. 1999, 20 , $217-230$ 